\title{
PTSD, Immunoglobulins, and Cortisol Changes after the Provision of the EMDR - PRECI to Females Patients with Cancer-Related PTSD Diagnosis
}

\author{
Benito Daniel Estrada ${ }^{1}$, , Bernardo de Jesús Angulo ${ }^{1,}$, , María Elena Navarro ${ }^{1}$, Ignacio Jarero ${ }^{2}$, \\ Omar Sánchez-Armass ${ }^{1}$ \\ ${ }^{1}$ Department of Research, San Luis Potosí Autonomous University, San Luis Potosí, Mexico \\ ${ }^{2}$ Department of Research, Mexican Association for Mental Health Support in Crisis, Mexico City, Mexico
}

\section{Email address:}

benito.estrada@uaslp.mx (B. D. Estrada), Valtiel23@live.com.mx (B. de J. Angulo), maelenanavarro@hotmail.com (M. E. Navarro), nacho@amamecrisis.com.mx (I. Jarero).marsac@gmail.com(O. Sánchez-Armass)

${ }^{*}$ Corresponding author

\section{To cite this article:}

Benito Daniel Estrada, Bernardo de Jesús Angulo, María Elena Navarro, Ignacio Jarero, Omar Sánchez-Armass. PTSD, Immunoglobulins, and Cortisol Changes after the Provision of the EMDR - PRECI to Females Patients with Cancer-Related PTSD Diagnosis. American Journal of Applied Psychology. Vol. 8, No. 3, 2019, pp. 64-71. doi: 10.11648/j.ajap.20190803.12

Received: June 2, 2019; Accepted: June 29, 2019; Published: July 13, 2019

\begin{abstract}
EMDR therapy administered to patients with cancer has proven to be effective in the reduction of posttraumatic stress disorder (PTSD) symptoms and PTSD diagnosis remission. The main objective of this study was to measure the PTSD scores and diagnosis changes before and after the provision of the EMDR-Protocol for Recent Critical Incidents and Ongoing Traumatic Stress (EMDR-PRECI) to female patients with cancer-related PTSD diagnosis. A secondary objective was to observe the immunoglobulins and the cortisol changes pre and post-treatment. Seven female patients with different types of cancer ( 5 breast, 1 colon, and 1 lymphatic cancer), different cancer treatments (radiation, chemotherapy, radiation, and chemotherapy), and PTSD diagnosis related to their diagnosis and cancer treatment met the inclusion criteria. Participants' age ranged from 40 to 57 years old ( $\mathrm{M}=52.28$ years old). For PTSD diagnosis and total scores, we used the Global Assessment of Posttraumatic Stress Questionnaire validated for the Mexican population. Biomarkers (cortisol and immunoglobulins) were measured before and after EMDR treatment. For the neuroendocrine measure, we used the cortisol levels in the participant's blood. For the immunological measure, we used the Nephelometry technique. We measured the changes in the following types of antibodies (immunoglobulins): a) Immunoglobulin A (IgA), b) Immunoglobulin G (IgG), and c) Immunoglobulin M (IgM). EMDR-PRECI was provided by three licensed EMDR clinicians formally trained in the protocol administration. Each EMDRPRECI session lasted 50-60 minutes. The minimum number of sessions was three and the maximum ten with an average of five. Treatment focused only on the distressing memories related to diagnosis and cancer treatment. No adverse effects were reported during treatment or at six months post-treatment assessment. Results showed a full PTSD diagnosis remission in all participants with significant differences for PTSD scores, $\mathrm{t}(6)=2.44, \mathrm{p}<.05$. These results are in concordance with Carletto et al., study in which all patients treated with EMDR no longer met criteria for PTSD [12]. No significant differences were found for immunoglobulins or cortisol. We believe that the administration of the EMDR-PRECI could be an efficient and effective component of a psychosocial approach to reduce or eliminate cancer-related PTSD symptoms and diagnosis.
\end{abstract}

Keywords: Eye Movement Desensitization and Reprocessing, PRECI, Cancer, Cortisol, Immunoglobulins, Posttraumatic Stress Disorder (PTSD)

\section{Introduction}

Cancer is the second leading cause of death globally and is estimated to account for 9.6 million deaths in 2018. Lung, prostate, colorectal, stomach and liver cancer are the most common types of cancer in men, while breast, colorectal, lung, cervix and thyroid cancer are the most common among women [1]. Cancer may involve stressful events that are 
repeated or continued over time. The patients with cancer may suffer cancer-related posttraumatic stress symptoms (PTSS) from diagnosis through treatment, after treatment, or during cancer recurrence [2]. Patients who have a history of posttraumatic stress disorder (PTSD) from previous trauma could have problems adjusting to cancer treatment [2].

PTSD is a mental condition characterized by a constellation of symptoms that occur following exposure to a traumatic event. Those symptoms include intrusion, avoidance of thoughts or reminders of the trauma, negative alterations in cognition and mood, and marked alterations in arousal and reactivity [3]. Literature examining PTSD symptomatology among long-term cancer survivors has found rates of lifetime cancer-related PTSD that range from $3 \%$ to $22 \%$, and the presence of comorbid psychiatric illnesses such as major depressive disorder and generalized anxiety disorder that may lead to worse survival outcomes [4]. Since 2008, the Institute of Medicine (IOM) has recommended that the standard of quality cancer care must now include services and interventions to address the psychosocial needs of cancer patients [5].

\subsection{EMDR Therapy}

Eye movement desensitization and reprocessing (EMDR) therapy and trauma-focused Cognitive Behavioral Therapy (TF-CBT) are the only psychotherapies recommended for children, adolescents, and adults with PTSD in the WHO Guidelines for the Management of Conditions Specifically Related to Stress [6, 7, 8]. EMDR therapy is a structured eight-phase comprehensive approach that addresses the past, present, and future aspects of distressing memories of adverse life experiences. It is guided by the Adaptive Information Processing (AIP) model [7]; which posits that memory networks are the basis of pathology and health. The AIP is the cornerstone of EMDR Therapy because it interprets clinical phenomena, predicts successful treatment outcomes, and guides clinical practice.

\subsection{EMDR Therapy Administered to Patients with Cancer}

\subsubsection{EMDR Therapy Provided in Individual Format}

In a controlled pilot study conducted by Capezzani et al., for patients with cancer and PTSD, patients treated with EMDR therapy had significant improvement 1-month posttreatment compared to the CBT group. Ten of the 11 patients treated with EMDR therapy recovered from their PTSD diagnosis compared to only one of the CBT patients [9].

In a nonrandomized study, Faretta et al., evaluated the effectiveness of EMDR therapy compared to a non-traumafocused CBT intervention in 57 (11 males and 46 females) participants with mixed cancer diagnoses that received 12 sessions of 60 minutes each. Those receiving EMDR therapy showed significantly larger posttreatment improvement, compared to the CBT group, on a number of measures, including traumatic stress, depression, and anxiety [10].

In this nonrandomized controlled trial thirty-seven women diagnosed with glioblastoma multiforme (GBM) received standard medical care. Of those, eighteen were treated during four months with 10-12 individual EMDR therapy sessions and nineteen were used as a nonrandomized control group. Results in the treatment group showed a significant decrease in anxiety, depression, and anger, as well a positive impact upon a sense of coherence level when compared to the control group [11].

In a quasi-experimental study, Carletto et al., aimed to evaluate the efficacy of EMDR therapy as compared to treatment as usual (TAU) in breast cancer (BC) patients with PTSD, identifying by electroencephalography (EEG) the neurophysiological changes underlying treatments effect and their correlation with clinical symptoms [12]. Thirty patients with BC and PTSD diagnosis were included, receiving either EMDR $(n=15)$ or TAU $(n=15)$. At post-treatment, all patients treated with EMDR no longer met criteria for PTSD, while all patients treated with TAU maintained the diagnosis. A significant decrease in depressive symptoms was found only in the EMDR group, while anxiety remained stable in all patients. EEG results corroborated these findings, showing a significant increase in neuronal synchronization in left angular and in right fusiform gyri only in the EMDR group. The efficacy of EMDR therapy in reducing cancerrelated PTSD is supported by both clinical and neurobiological findings.

\subsubsection{EMDR Therapy Provided in Group Format}

Jarero et al., conducted a pilot study to evaluate the effectiveness of EMDR-Integrative Group Treatment Protocol Adapted for Ongoing Traumatic Stress (EMDRIGTP-OTS) in reducing cancer-related posttraumatic stress disorder (PTSD) for adult women [13]. EMDR intensive therapy was administered for three consecutive days, twice daily, to 24 adult women diagnosed with different types of cancer (cervical, breast, colon, bladder, and skin) who had PTSD symptoms related to their diagnosis and treatment. Treatment outcomes were compared between patients in the active phase of cancer treatment and those in the follow-up phase, with scores on the Short PTSD Rating Interview (SPRINT) at pre- and post-EMDR treatment and at 30- and 90-day follow-up [14, 15]. Results showed no difference between groups, with significant improvement in both groups for PTSD symptoms and overall subjective well-being.

To extend the previous investigation, Jarero et al., conducted a randomized controlled trial (RCT) on the provision of the EMDR-IGTP-OTS to 65 female adults' patients with different types of cancer and cancer-related PTSD symptoms [16]. A comparison of the treatment and notreatment control groups showed a significantly greater decrease for the treatment group on symptoms of PTSD measured with the Posttraumatic Stress Disorder Checklist for DSM-5 (PCL-5) [17]; anxiety and depression symptoms measured with the Hospital Anxiety and Depression Scale (HADS) in the pre-post and 90-days follow-up measurements [18], suggesting that the EMDR-IGTP-OTS may be an efficient and effective way to address cancer-related 
posttraumatic, depressive, and anxious symptoms.

Osorio et al., conducted a randomized controlled trial to evaluate the effectiveness of the EMDR-IGTP-OTS in reducing posttraumatic stress disorder symptoms, depression and anxiety symptoms related to the diagnosis and treatment of cancer in adolescents and young adults [19]. Twenty-three adolescents and young adults (13 male and 10 female) with different types of cancer (breast, leukemia, lymphoma) and PTSD symptoms related to their diagnosis and cancer treatment met the inclusion criteria. Participants age ranged from 13 to 22 years old $(\mathrm{M}=16.71$ years). Participants in treatment $(\mathrm{N}=11)$ and no-treatment control $(\mathrm{N}=12)$ groups completed pre, post, and follow up measurements using the PCL- 5 and the HADS. Data analysis by repeated measures ANOVA showed that the EMDR-IGTP-OTS was effective in significantly reducing symptoms of PTSD, anxiety, and depression, with symptoms maintained at 90-day follow-up and with large effect sizes (e.g., $d=1.17$ ). This study suggests that EMDR-IGTP-OTS may be an efficient and effective way to address cancer-related PTSD, depressive, and anxious symptoms in adolescents and young adults.

Roberts conducted a pre-experimental case study to explore the efficacy and safety of the EMDR Group Traumatic Episode Protocol (G-TEP) on 35 cancer survivors with various types of cancers in different stages [20, 21]. Participant's received two 90-minute G-TEP sessions, administered in consecutive days. Repeated measures comparison of PTSD symptoms revealed some significant differences in anxiety and depression over time, and modest changes across the entire sample between posttest and follow-up. Results support the need for research with large samples and randomized clinical trials to examine the viability of providing G-TEP to a cancer survivor.

\subsection{EMDR-PRECI}

The EMDR Protocol for Recent Critical Incidents and Ongoing Traumatic Stress (EMDR-PRECI) is an eight-phase and three-pronged protocol developed in the field to treat recent (even hours after a trauma) or prolonged adverse experiences where related stressful events continue for an extended time and where there is no post-trauma safety period for traumatic memory consolidation [22]. Shapiro recommends the EMDR-PRECI "for an extended postdisaster period to address situations in which there is ongoing trauma and therefore no subsequent period of safety" [7] (p. 397).

EMDR-PRECI conceptualizes the adverse experience as an extended event, with a continuum of ongoing external stressful events with similar emotions and physical sensations, interfering with memory consolidation and creating a cumulative trauma exposure memory network of linked pathogenic memories with similar emotional, somatic, sensorial, and cognitive information, that extends into the present moment, and often producing maladaptive/catastrophic concerns about the future or flashforward [23-26]. For more information about this protocol, see Jarero, Artigas, and Luber [22].

\subsection{EMDR-PRECI Previous Studies}

In this randomized controlled trial (RCT) using a delayed treatment-controlled design, 18 adults were treated within 30 days following a 7.2 earthquake in North Baja California, Mexico in 2010 using a single (80- to 130-minute) session of EMDR-PRECI [22]. Despite frequently occurring aftershocks, both groups (immediate-treatment group and delayed-treatment control group) showed substantial (30 points) reductions of trauma symptoms on the Impact of Events Scale [27], effects that were maintained at a 12-week follow-up. No participant developed PTSD during this period.

In this delayed treatment control design, a single EMDRPRECI individual session (90-120 minutes) was provided within 8 weeks post-event to 32 first responder forensic personnel who were collecting body parts following a massacre of hundreds of people [28, 29]. Results showed significant improvement for both immediate treatment $(\mathrm{N}=$ $18)$ and waitlist/delayed treatment $(\mathrm{N}=14)$ groups on the Impact of Events Scale and Short PTSD Rating Interview (SPRINT) [27, 14, 15]. Follow-up scores at 3- and 5-months posttreatment showed that the original treatment results were maintained, with a further significant reduction of selfreported symptoms of posttraumatic stress and PTSD between posttreatment and follow-up. This study suggests that EMDR-PRECI was an effective early intervention, reducing traumatic stress for a group of traumatized adults continuing to work under extreme stressors in a human massacre situation. It appears that the treatment may have helped to prevent the development of chronic PTSD and to increase psychological and emotional resilience.

In this RCT an adaptation of the EMDR-PRECI for paraprofessionals use in acute trauma situations named the EMDR Individual Protocol for Paraprofessional use (EMDRPROPARA) was provided to 39 traumatized first responders on active duty randomly assigned to receive two 90-minute sessions of either EMDR-PROPARA or of supportive counseling [24]. Participants in the EMDR-PROPARA group showed benefits immediately after treatment, with their scores on the SPRINT showing further decreases at 3-month follow-up. In comparison, supportive counseling participants experienced a nonsignificant decrease after treatment and an increase in the SPRINT scores at the second follow-up. The significant difference between the two treatments provides preliminary support for EMDR-PROPARA's effectiveness in reducing the severity of posttraumatic symptoms and subjective global improvement.

In this RCT using a delayed treatment-controlled design, the EMDR-PRECI was administered within 34 days of an explosion in an explosives manufacturing factory in Mexico that killed seven employees [30]. Twenty-five survivors who had posttraumatic stress symptoms related to the critical incident received two 60-minute sessions on consecutive days. Initial scores for both groups were in the severe range for trauma symptoms, as measured by the SPRINT and decreased to low levels after treatment (from 22 to 2). 
Treatment effects were maintained 106 days after the explosion, and no participant developed PTSD during this period.

A randomized controlled clinical trial evaluated the effectiveness of the EMDR-PRECI in PTSD, anxiety, and depression symptoms in family caregivers of patients with autism spectrum disorder (ASD) [31]. Participants were randomly assigned to six 60-minute individual treatment sessions $(\mathrm{N}=14)$ or to care-as-usual control condition $(\mathrm{N}=$ 12). They completed pre, post, and follow up measurements PCL-5 and HADS, while participants continued taking care of their relatives with ASD, during the follow-up. The study results suggest that the EMDR-PRECI could be an efficient and effective treatment to address family caregiver's PTSD, anxiety, and depression symptoms.

In this RCT 60 first responders were randomly assigned to two 60-minute individual treatment sessions $(\mathrm{N}=30)$ or a no-treatment control condition $(\mathrm{N}=30)$ [32]. They completed pre, post, and follow up measurements using the PCL-5 and the HADS. Data analysis by repeated measures ANOVA showed clear effects of the EMDR-PRECI in reducing PTSD work-related symptoms in the treatment group with symptom reduction maintained at 90-day followup with a large effect size $(\mathrm{d}=3.99)$, while participants continued to experience direct exposure to potentially traumatic work-related events during the follow-up period. The study results suggest that the EMDR-PRECI could be an efficient and effective way to address first responders' workrelated PTSD, anxiety, and depression symptoms.

\section{Objective}

The main objective of this study was to measure the PTSD scores and diagnosis changes before and after the provision of the EMDR-PRECI to female patients with cancer-related PTSD diagnosis. A secondary objective was to observe the immunoglobulins and the cortisol changes pre and posttreatment.

\section{Method}

\subsection{Study Design}

To measure the PTSD scores, immunoglobulins and cortisol changes before and after the provision of the EMDRPRECI, this study used a pre-treatment-post-treatment measurement design.

\subsection{Ethics}

The research protocol was reviewed and approved by the Institutional Review Board of the Central Hospital "Ignacio Morones Prieto" from San Luis Potosí, Mexico, and was registered with the number 73-16 in September 2016.

\subsection{Participants}

This study was conducted during 2017-2018 in the city of San Luis Potosí, Mexico. Potential participants were recruited through social networks or other patients with cancer. People interested were asked to call by phone to receive the details of the research project. A total of 50 potential participants called and received the information. Of those, 10 were able to attend the personal interview. The other 40 could not find the time to attend the interview. Seven persons that attended the intake interview fulfilled the inclusion criteria.

Inclusion criteria were: (a) Female adult, (b) diagnosis of cancer, (c) be in the active or follow-up phase of cancer treatment, (d) have received or been receiving treatment (e.g., surgery, radiotherapy and/or chemotherapy), (e) with PTSD diagnosis related to their diagnosis and cancer treatment, (f) without acute infections, (g) not receiving specialized trauma therapy, (h) not receiving drug therapy for the posttraumatic stress disorder symptoms.

Exclusion criteria were: (a) ongoing immunology treatment, (b) be in terminal phase, (c) ongoing viral, autoimmune or allergic disease, (d) ongoing selfharm/suicidal or homicidal ideation, (e) diagnosis of psychotic or bipolar disorder, (f) diagnosis of dissociative disorder, (g) organic mental disorder, (h) current, active chemical dependency problem, (i) significant cognitive impairment (e.g., severe intellectual disability, dementia).

Seven female patients with different types of cancer (5 breast, 1 colon, and 1 lymphatic cancer), different cancer treatments (radiation, chemotherapy, radiation, and chemotherapy), and PTSD diagnosis related to their diagnosis and cancer treatment met the inclusion criteria. Participants' age ranged from 40 to 57 years old $(\mathrm{M}=52.28$ years old).

\subsection{Instrument for PTSD and Biomarkers Techniques}

1) For PTSD diagnosis and total scores, we used the Global Assessment of Posttraumatic Stress Questionnaire (GAPSQ) [33]. It is a PTSD diagnostic instrument for adults based on the Diagnostic and Statistical Manual of Mental Disorders, Text Revised $4^{\text {th }}$ edition (DSM-IV-TR) [34]. It has 62 items divided into three sections that refer to the evaluation of traumatic events, symptomatology, and functioning of the person. The Cronbach alpha reported for the Spanish population was 0.92 . Psychometric validation of this instrument was conducted for the Mexican population [35]. Results were similar to those obtained in the Spanish population. The total scale showed a very good internal consistency with a Cronbach alpha coefficient of 0.93 . Therefore, it is a valid and reliable instrument to evaluate PTSD in the Mexican population.

2) For the neuroendocrine measure, we used the cortisol levels in the participant's blood. For the Immunological measure, we used the Nephelometry technique. This technique is used in immunology to determine the levels of some blood plasma proteins. It is performed by measuring the turbidity in a water sample by passing laser light through the sample being measured. With this technique, we measured the changes in the following types of antibodies (immunoglobulins): a) Immunoglobulin A (IgA). This is an 
antibody that plays a crucial role in the immune function of mucous membranes and represents up to $15 \%$ of total immunoglobulins produced throughout the body; b) Immunoglobulin $\mathrm{G}$ (IgG). This is the most common type of antibody found in blood circulation and represents approximately $75 \%$ of serum (blood plasma) antibodies in humans, and c) Immunoglobulin M (IgM). This is the largest antibody, and it is the first antibody to appear in response to initial exposure to an antigen. The spleen is the major site of specific IgM production.

\subsection{Procedure}

\subsubsection{Biomarkers Collection Procedure, PTSD, and Confidentiality of Data}

For the immunoglobulins and cortisol changes, the blood samples were collected between 6:00 A. M. and 8:00 A. M., with a minimum of six hours of fasting. Patients did not have an acute infection due to fungi, bacteria, or viruses at the time of the analysis. In the laboratory, sterile instruments were used, and the flasks with the blood sample were deprived of strong light. The post-treatment assessment was conducted at six months.

For PTSD diagnosis and total scores, participants completed the GAPSQ on an individual basis in different measurement moments. The post-treatment assessment was conducted at six months. All data was collected, stored, and handled in full compliance with the Central Hospital "Ignacio Morones Prieto" Institutional Review Board to ensure confidentiality. Each study participant gave their consent for access to their personal data, which was strictly required for study quality control. All persons involved in this research project are subject to professional confidentiality.

\subsubsection{Withdrawal from the Study}

All research participants had the right to withdraw from the study without justification at any time and with assurances of no prejudicial result. If participants decided to withdraw from the study, they were no longer followed up in the research protocol. There were no withdrawals from this study.

\subsubsection{Treatment}

\section{Therapists and Treatment Fidelity}

EMDR-PRECI was provided by three licensed EMDR clinicians formally trained in the protocol administration. Treatment fidelity and adherence to the protocol was fulfilled by EMDR therapists' strict observance to all steps of the scripted protocol.

\section{EMDR-PRECI Treatment Description}

Each EMDR-PRECI session lasted 50-60 minutes. The minimum number of sessions was three and the maximum ten with an average of five. Treatment focused only on the distressing memories related to diagnosis and cancer treatment. To encompass the whole ongoing traumatic stress spectrum, clinicians asked each of the participants to remember the worst event that they selected during the GAPSQ assessment. Once the clients had reprocessed the worst event, clinicians asked them to run a mental movie of the whole cancer-related experience from right before the beginning until today, or even into the future, and then to identify any other disturbing parts/memories. All participants reprocessed more than one memory. No adverse effects were reported during treatment or at six months post-treatment assessment.

Laboratory and PTSD Pre and Post Treatment Results

On Tables 1, 2 and 3 we can see the pre-treatment and post-treatment immunoglobulins $\operatorname{IgA}, \operatorname{IgG}$, and $\operatorname{IgM}$ measurements. On Table IV, we can see the pre-treatment and post-treatment cortisol measurement. On Table V, we can see the pre-treatment and post-treatment GAPSQ PTSD symptoms scores and diagnosis.

Table 1. Immunoglobulin IgA.

\begin{tabular}{|c|c|c|c|c|}
\hline & & PRE (I. C.: 99.9\%) & POST (I. C.: 99.9\%) & \\
\hline \multirow{7}{*}{$\operatorname{IgA}$} & S1 & $143.1(143.007-143.103)$ & $158.2(158.197-158.203)$ & \multirow{7}{*}{$71-360$} \\
\hline & S2 & $180.4(180.397-180.403)$ & $180.8(180.797-180.803)$ & \\
\hline & S3 & $146.8146 .797-147.803)$ & $133.7(133.697-133.703)$ & \\
\hline & S4 & $120.2(120.197-120.203)$ & $117.0(116.997-117.003)$ & \\
\hline & S5 & $36.3(36.297-36.303)$ & $64.7(64.697-64-703)$ & \\
\hline & S6 & $229.2(229.197-229.203)$ & $207.1(207.097-207.103)$ & \\
\hline & S7 & $228.5(228.497-228.503)$ & $154.3(154.297-154.303)$ & \\
\hline
\end{tabular}

Table 2. Immunoglobulin $\operatorname{Ig} G$.

\begin{tabular}{lllll}
\hline & & PRE (I. C.: 99.9\%) & POST (I. C.: 99.9\%) \\
\hline & S1 & $878.4(878.397-878.403)$ & $1181(1180.997-1181.003)$ & $763.8(763.797-763.803)$ \\
IgG & S2 & $1003(1002.997-1003.003)$ & $919.8(919.797-919.803)$ & $658-1837$ \\
& S3 & $900.6(900.597-900.603)$ & $515.1(515.097-515.103)$ & \\
& S4 & $600.3(600.297-600.303)$ & $887.6(887.597-887.603)$ & \\
& S5 & $970.3(970.297-970.303)$ & $744.5(744.497-744.503)$ & \\
& S6 & $869.5(869.497-869.503)$ & $856.0(855.997-856.003)$ & \\
\hline
\end{tabular}


Table 3. Immunoglobulin IgM.

\begin{tabular}{|c|c|c|c|c|}
\hline & & PRE (I. C.: 99.9\%) & POST (I. C.: 99.9\%) & \\
\hline \multirow{7}{*}{$\operatorname{IgM}$} & S1 & $56.4(56.397-56.403)$ & $59.8(59.797-59.803)$ & \multirow{7}{*}{$40-263$} \\
\hline & S2 & $105.2(105.197-105.203)$ & $123.2(123.197-123.203)$ & \\
\hline & S3 & $123.1(123.097-123.103)$ & $97.2(97.197-97.203)$ & \\
\hline & S4 & $54.3(54.297-54.303)$ & $26.5(26.497-26.503)$ & \\
\hline & S5 & $167.4(167.397-167.403)$ & $66.8(66.797-66.803)$ & \\
\hline & S6 & $177.3(177.297-177.303)$ & $92(91.997-92.003)$ & \\
\hline & S7 & $85.9(85.897-85.903)$ & $48(47.997-48.003)$ & \\
\hline
\end{tabular}

Table 4. Cortisol.

\begin{tabular}{|c|c|c|c|c|}
\hline & & PRE (I. C.: 99.9\%) & POST (I. C.: 99.9\%) & \\
\hline \multirow{7}{*}{ Cortisol } & S1 & $11.0(10.997-11.003)$ & $10.7(10.697-10.703)$ & \multirow{7}{*}{$5-25$} \\
\hline & $\mathrm{S} 2$ & $9.79(9.787-9.793)$ & $8.53(8.527-8.533)$ & \\
\hline & S3 & $8.59(8.587-8.593)$ & $11.7(11.697-11.703)$ & \\
\hline & S4 & $8.83(8.827-8.833)$ & $11.0(10.997-11.003)$ & \\
\hline & S5 & $18.2(18.197-18.202)$ & $17.7(17.697-17.703)$ & \\
\hline & S6 & $6.97(6.967-6.973)$ & $11.4(11.397-11.403)$ & \\
\hline & S7 & $12.9(12.897-12.903)$ & $10.6(10.597-10.603)$ & \\
\hline
\end{tabular}

Table 5. GAPSQ PTSD Scores and Diagnosis.

\begin{tabular}{lll}
\hline Patient & Pre-treatment scores and diagnosis & Post-treatment scores and diagnosis \\
\hline 1 & 30 and full PTSD & 8 and PTSD full remission \\
2 & 81 and full PTSD & 10 and PTSD full remission \\
3 & 25 and full PTSD & 1 and PTSD full remission \\
4 & 54 and full PTSD & 8 and PTSD full remission \\
5 & 26 and full PTSD & 12 and PTSD full remission \\
6 & 44 and full PTSD & 16 and PTSD full remission \\
7 & 33 and full PTSD & 5 and PTSD full remission \\
\hline
\end{tabular}

\section{Statistical Analysis and Results}

T-test was applied to compare means in pre-treatment and post-treatment of the paired sample. Results showed significant differences for PTSD scores, $\mathrm{t}(6)=2.44, \mathrm{p}<.05$. No significant differences were found for immunoglobulins or cortisol. See Table 6.

Table 6. Mean scores and standard deviations for PTSD, Immunoglobulins, and Cortisol on pre-treatment and post-treatment measurements $(N=7)$.

\begin{tabular}{lllll}
\hline \multirow{2}{*}{ Variable } & Time 1 & \multicolumn{3}{l}{ Time 2 } \\
\cline { 2 - 5 } & M & SD & M & SD \\
\hline Posttraumatic Stress (GAPSQ) & 41.85 & 20.16 & 8.57 & 4.82 \\
IgA & 154.92 & 67.15 & 145.11 & 46.11 \\
IgG & 898.01 & 149.24 & 838.14 & 202.28 \\
IgM & 109.94 & 49.28 & 73.35 & 32.82 \\
Cortisol & 10.85 & 3.73 & 11.70 & 2.83 \\
\hline
\end{tabular}

\section{Discussion}

The main objective of this study was to measure the PTSD scores and diagnosis changes before and after the provision of the EMDR-PRECI to female patients with cancer-related PTSD diagnosis. A secondary objective was to observe the immunoglobulins and the cortisol changes pre and posttreatment. Seven female patients with different types of cancer ( 5 breast, 1 colon, and 1 lymphatic cancer), different cancer treatments (radiation, chemotherapy, radiation, and chemotherapy), and PTSD diagnosis related to their diagnosis and cancer treatment met the inclusion criteria. Results showed a full PTSD diagnosis remission in all participants with significant differences for PTSD scores, $t$ (6) $=2.44, \mathrm{p}<.05$. These results are in concordance with Carletto et al., study in which all patients treated with EMDR no longer met criteria for PTSD [12]. No significant differences were found for immunoglobulins or cortisol. The discussion of the results of the biomarker is beyond the scope of this study. In addition to physical suffering, patients with cancer may develop PTSD. Up to $35 \%$ of patients with cancer suffer from cancer-related posttraumatic symptoms [36, 37]. Examples of this symptoms are flashbacks, sense of 
shortened future, nightmares, difficulty sleeping, fears of recurrence, restlessness, avoidant behavior toward places (e.g., hospitals, chemotherapy setting), and persons related to their cancer experience. Intrusive symptoms (i.e., repeated, disturbing, and unwanted memories of the cancer experience, repeated disturbing dreams of the cancer experience, suddenly feeling or acting as if the cancer experience were actually happening again, feeling very upset when something reminds you the cancer experience) appear to be the core symptoms in oncology patients [38]. When cancer-related PTSD is untreated, treatment nonadherence, pain, desire to die, and disability may increase [39]. Due to improvements in cancer care, survival rates are increasing, and relief from physical and psychosocial impact is an extremely important component of cancer care for enhancing the quality of life [40].

\section{Conclusion, Limitations, and Future Directions}

Cancer is a unique and not time-limited stressor. The cumulative trauma exposure memory network of linked pathogenic memories formed by multiple and prolonged traumatic experiences (diagnosis, surgery, mutilation, noxious treatments, progressive deterioration) distinguish cancer from a discrete, time-limited traumatic event. Therefore, it is important for mental health professionals to assess for the presence of cancer-related PTSD and offer cancer-related PTSD evidence-based therapeutic interventions to improve cancer patients' quality of life. We believe that the administration of the EMDR-PRECI could be an efficient and effective component of a psychosocial approach to reduce or eliminate cancer-related PTSD symptoms and diagnosis.

This study has several limitations, notably the pretreatment-post-treatment measurement design without a control group and the small sample size. Future research with randomized controlled clinical trials with larger samples with different types of cancer, and using more biomarkers (e.g., epinephrine, norepinephrine, cytotoxicity levels of natural killer cells) to measure the immune response and neuroendocrine changes is highly recommended.

\section{Conflict of Interest and Founding}

The authors have no relevant financial interest or affiliation with any commercial interest related to the subjects discussed in this article. This study was founded with resources from the Mexican Government Department of Education and from the non-governmental organization (NGO) Patronato Pro Paciente Oncológico, A. C., San Luis Potosí, México.

\section{Acknowledgements}

We want to express gratitude to the following individuals for their contributions: Martha Givaudan, and Lynne Levinson.

\section{References}

[1] World Health Organization (2019). Cancer. Retrieved May 21, 2019 from https://www.who.int/cancer/en/.

[2] National Cancer Institute (2019). Cancer-related posttraumatic stress (PDQ ${ }^{\circledR}$ )-Patient Version. Retrieved May 21, 2019 from https://www.cancer.gov/about-cancer/coping/survivorship/newnormal/ptsd-pdq.

[3] American Psychiatric Association. (2013). Diagnostic and statistical manual of mental disorders (5th ed.). Arlington, VA.

[4] Andrykowski, M. A., \& Kangas, M. (2010). Posttraumatic stress disorder associated with cancer diagnosis and treatment. In J. C. Holland, W. S. Breitbart, P. B. Jacobsen, M. S. Lederberg, M. J. Loscalzo, \& R. McCorklen (Eds.), Oxford textbook of psycho-oncology (2nd ed., pp. 348-357). New York, NY: Oxford University Press.

[5] Institute of Medicine (2008). Committee on Psychosocial Services to Cancer Patients/Families in a Community Setting, Adler NE, Page A, eds. Cancer Care for the Whole Patient: Meeting Psychosocial Health Needs. Washington, DC: National Academies Press.

[6] Shapiro, F. (2001). Eye movement desensitization and reprocessing. Basic principles, protocols, and procedures (2nd ed.). New York, NY: Guilford Press.

[7] Shapiro, F. (2018). Eye movement desensitization and reprocessing. Basic principles, protocols, and procedures (Third edition). Guilford Press.

[8] World Health Organization. (2013). Guidelines for the management of conditions specifically related to stress. Geneva, Switzerland: Author.

[9] Capezzani, L., Ostacoli, L., Cavallo, M., Carletto, S., Fernandez, I., Solomon, R.,. Cantelmi, T. (2013). EMDR and CBT for cancer patients: Comparative study of effects on PTSD, anxiety, and depression. Journal of EMDR Practice and Research, 7 (3), 134-143. http://dx.doi.org/10.1891/1933-3196.7.3.134.

[10] Faretta, E., Borsato, T., Civilotti, C., Fernandez, I., \& Pagani, M. (2016). EMDR and CBT: A comparative clinical study with oncological patients. Journal of EMDR Practice and Research, 10 (3), 215-227. http://dx.doi.org/10.1891/19333196.10.3.215.

[11] Szpringer, M., Oledzka, M., and Amann, B. L. (2018) A Nonrandomized Controlled Trial of EMDR on Affective Symptoms in Patients With Glioblastoma Multiforme. Front. Psychol. 9: 785. doi: 10.3389/fpsyg.2018.00785.

[12] Sara Carletto, Camillo Porcaro, Carmen Settanta, Vincenzo Vizzari, Maria Rosa Stanizzo, Francesco Oliva, Riccardo Torta, Isabel Fernandez, Mario Coletti Moja, Marco Pagani \& Luca Ostacoli (2019). Neurobiological features and response to eye movement desensitization and reprocessing treatment of posttraumatic stress disorder in patients with breast cancer, European Journal of Psychotraumatology, 10: 1, 1600832, DOI: $10.1080 / 20008198.2019 .1600832$. 
[13] Jarero, I., Artigas, L., Uribe, S., García, L. E., Cavazos, M. A., \& Givaudan, M. (2015). Pilot research study on the provision of the EMDR integrative group treatment protocol with female cancer patients. Journal of EMDR Practice and Research, 9 (2), 98-105.

[14] Connor, K. M., \& Davidson, J. R. (2001). SPRINT: A brief global assessment of post-traumatic stress disorder. International Clinical Psychopharmacology, 16 (5), 279-284.

[15] Vaishnavi, S., Payne, V., Connor, K., Davidson, J. R. (2006). A Comparison of the SPRINT and CAPS assessment scales for posttraumatic stress disorder. Depression and Anxiety, 23 (7), 437-440.

[16] Jarero, I., Givaudan, M., Osorio, A. Randomized Controlled Trial on the Provision of the EMDR Integrative Group Treatment Protocol Adapted for Ongoing Traumatic Stress to Female Patients with Cancer-Related Posttraumatic Stress Disorder Symptoms. (2018). Journal of EMDR Practice and Research, 12 (3), 94-104.

[17] Weathers, F. W., Litz, B. T., Keane, T. M., Palmieri, P. A., Marx, B. P., \& Schnurr, P. P. (2013). The PTSD Checklist for DSM-5 (PCL-5). Scale available from the National Center for PTSD at www.ptsd.va.gov.

[18] Zigmond, A. S. \& Snaith, R. P. (1983). The Hospital Anxiety and Depression Scale. Acta Psychiatrica Scandinavica, 67, 361-370.

[19] Osorio, A., Pérez, M. C., Tirado, S. G., Jarero, I., Givaudan, M. (2018). Randomized Controlled Trial on the EMDR Integrative Group Treatment Protocol for Ongoing Traumatic Stress with Adolescents and Young Adults Patients with Cancer. American Journal of Applied Psychology, 7 (4), 5056. doi: 10.11648/j.ajap. 20180704.11.

[20] Roberts, A. (2018). The effects of the EMDR Group Traumatic Episode Protocol with cancer survivors. Journal of EMDR Practice and Research, 12 (3), 105-117.

[21] Shapiro, E. "The EMDR Group Traumatic Episode Protocol." (2013). Presentation to the EMDR Turkey Conference, Istanbul, Turkey.

[22] Jarero, I., Artigas, L., \& Luber, M. (2011). The EMDR protocol for recent critical incidents: Application in a disaster mental health continuum of care context. Journal of EMDR Practice and Research, 5 (3), 82-94.

[23] Jarero, I., \& Artigas, L. (2018). AIP-model Based Acute Trauma and Ongoing Traumatic Stress Theoretical Conceptualization. Iberoamerican Journal of Psychotraumatology and Dissociation, 10 (1), 1-10. http://revibapst.com.

[24] Jarero, I., Amaya, C., Givaudan, M., Miranda, A. (2013). EMDR Individual protocol for paraprofessional use: A randomized controlled trial with first responders. Journal of EMDR Practice and Research, 7 (2), 55-64.

[25] Centonze, D., Siracusane, A., Calabresi, P., and Bernardi, G. (2005). Removing pathogenic memories. Mol. Neurobiol. 32, 123-132. doi: 10.1385/MN: 32: 2: 123,

[26] Logie, R., \& de Jongh, A. (2014). The flashforward procedure: Confronting the catastrophe. Journal of EMDR Practice and Research, 8, 25-32.
[27] Horowitz, M., Wilmer, N., \& Alvarez, W. (1979). Impact of Event Scale: A measure of subjective stress. Psychosomatic Medicine, 41 (3), 209-218.

[28] Jarero, I., \& Uribe, S. (2011). The EMDR protocol for recent critical incidents: Brief report of an application in a human massacre situation. Journal of EMDR Practice and Research, 5 (4), 156-166.

[29] Jarero, I., \& Uribe, S. (2012). The EMDR protocol for recent critical incidents: Follow-up Report of an application in a human massacre situation. Journal of EMDR Practice and Research, 6 (2), 50-61.

[30] Jarero, I., Uribe, S., Artigas, L., Givaudan, M. (2015). EMDR protocol for recent critical incidents: A randomized controlled trial in a technological disaster context. Journal of EMDR Practice and Research, 9 (4), 166-173. DOI: http://dx.doi.org/10.1891/1933-3196.9.4.166.

[31] Encinas, M., Osorio, A., Jarero, I., Givaudan, M. (2019). Randomized Controlled Clinical Trial of the Provision of the EMDR-PRECI to Family Caregivers of Patients with Autism Spectrum Disorder. Psychology and Behavioral Science International Journal, 11 (1), 1-8, DOI: 10.19080/PBSIJ.2019.11.555802.

[32] Jarero, I., Schnaider, S., Givaudan, M. (2019). EMDR Protocol for Recent Critical Incidents and Ongoing Traumatic Stress with First Responders: A Randomized Controlled Trial. Journal of EMDR Practice and Research, 13 (2).

[33] Crespo, M. \& Gómez, M. M. (2012). Escala Global de Estrés Postraumático (EGEP) [Posttraumatic Global Stress Scale; PGSS]. Madrid: TEA Ediciones.

[34] American Psychiatric Association. (2000). Diagnostic and statistical manual of mental disorders (4th ed., Text Rev.). Washington, DC: Author.

[35] Estrada, Delgado \& Sanchez-Prada (2016) Validation of the Global Assessment of Posttraumatic Stress Questionnaire overall general Mexican population. Unpublished manuscript.

[36] National Cancer Institute. (2015). Post-traumatic stress disorder. Retrieved from http://www.cancer.gov/cancertopics/pdq/supportivecare/posttraumatic-stress/Patient.

[37] Kangas, M., Henry, J., \& Bryant, R. A. (2002). Posttraumatic stress disorder following cancer: A conceptual and empirical review. Clinical Psychology Review, 22, 499-524.

[38] Carletto, S., Pagani, M. (2016). Neurobiological Impact of EMDR in Cancer. Journal of EMDR Practice and Research, $13(3), 153,161$.

[39] Kadan-Lottick NS, Vanderwerker LC, Block SD, et al. (2005). Psychiatric disorders and mental health service use in patients with advanced cancer. Cancer. 104: 2872-81. [PubMed: 16284994].

[40] French-Rosas, L., Moye, J., Naik, A. D. (2011). Improving the Recognition and Treatment of Cancer-Related Posttraumatic Stress Disorder. J Psychiatr Pract. 2011 July; 17 (4): 270-276. doi: 10.1097/01.pra.0000400264.30043.ae. 